\title{
Thalassemia and spinal cord compression in pregnancy
}

\author{
Mohsen Esfandbod MD, Mahdi Malekpour MD
}

Previously published at www.cmaj.ca

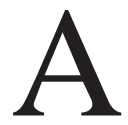

22-year-old nulliparous woman in her 32nd week of pregnancy was referred to our emergency department because she had been unable to walk for three days. Three weeks before admission, she had felt a burning sensation in her lower thoracic region, which she attributed to the pregnancy. However, she had increasing difficulty in walking and standing and eventually became bedridden. Urination and defecation were normal. Three years before admission, she had been diagnosed with thalassemia intermedia with a hemoglobin level of 100 (normal 124-146) g/L and a fetal hemoglobin level of $60 \%$, but had never required transfusion.

On examination, the patient had sinus tachycardia, and her skin and mucous membranes were pale. Uterine size was appropriate for a 32-week pregnancy. Neurologic examination was consistent with spastic paraplegia. Power was $3 / 5$ in her right leg and 2/5 in her left leg. Her deep tendon reflexes were increased, plantar reflexes were upward, and clonus was detected in her legs (see video in Appendix 1, available at www.cmaj.ca/cgi/content/full/cmaj.091427/DC1). She had decreased sensation below the T10 level. Anal sphincter tone was normal. Her hemoglobin level had dropped to $80 \mathrm{~g} / \mathrm{L}$.

The patient was given dexamethasone for spinal cord syndrome and underwent emergency magnetic resonance imaging (MRI) of her spine. A posterior extradural mass, suggestive of an extramedullary hematopoietic centre, compressing the spinal cord was seen from levels T4 to T8 (Figure 1A). A presumptive diagnosis of extramedullary hematopoiesis was made. Because of the high risk of bleeding, a biopsy of the mass was not undertaken. An emergency cesarean delivery was performed under general anesthesia, resulting in the successful delivery of a healthy infant. The patient underwent radiotherapy and concomitant treatment with hydroxyurea for 14 days, along with hypertransfusion to reach a hemoglobin level of $150 \mathrm{~g} / \mathrm{L}$. Follow-up MRI after 30 days showed no sign of the mass (Figure 1B) and the patient could walk without difficulty. There were no major neurologic sequelae.

Extramedullary hematopoiesis, defined as the formation and development of blood cells outside the bone marrow, can occur to compensate for insufficient production of blood elements in hematologic disorders, such as leukemia, myelofibrosis and hereditary hemoglobinopathy. ${ }^{1}$ It is observed in $30 \%-60 \%$ of

From the Departments of Hematology and Oncology (Esfandbod), and Obstetrics and Gynecology (Esfandbod, Malekpour), Imam Khomeini Hospital, Tehran University of Medical Sciences, Tehran, Iran

CMAJ 2010. DOI:10.1503/cmaj.091427

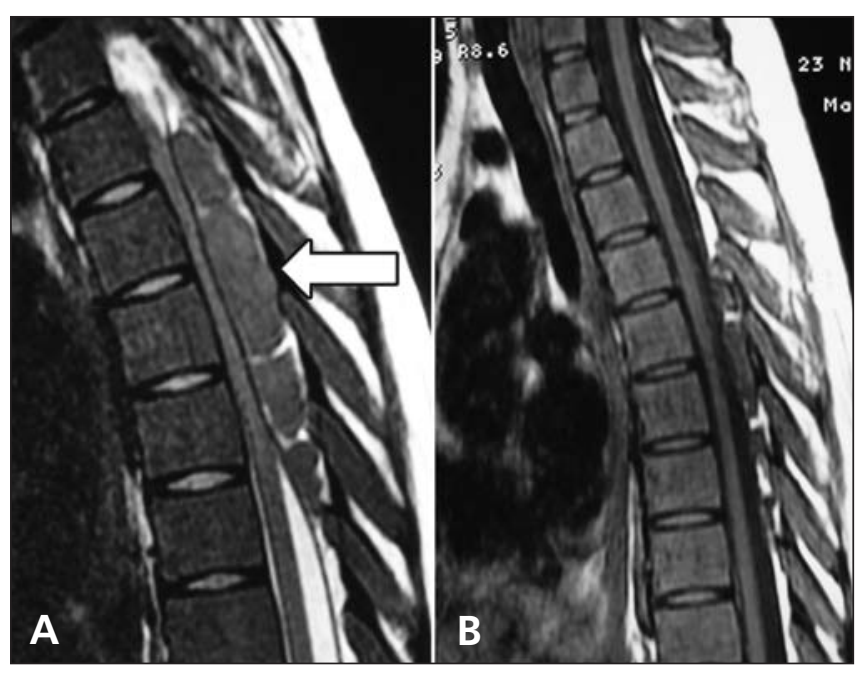

Figure 1: Magnetic resonance image of the dorsal spine in a 22year-old pregnant woman with thalassemia intermedia and spastic paraplegia. (A) A posterior extradural mass suggestive of extramedullary hematopoisis (arrow) compressing the spinal cord. (B) A follow-up image from one month later shows no mass.

patients with thalassemia intermedia. ${ }^{2}$ The liver, spleen and lymph nodes are common sites for extramedullary hematopoiesis. ${ }^{3}$ Three main treatment options include surgical excision, radiotherapy and hypertransfusion. ${ }^{2}$ Extramedullary hematopoiesis is almost always asymptomatic, but rarely compression of affected organs provokes clinical manifestations. The condition is diagnosed early and treated successfully in most patients, making poor outcomes uncommon. ${ }^{4}$

This article has been peer reviewed.

Competing interests: None declared.

\section{REFERENCES}

1. Weatherall DJ, Provan AB. Red cells I: inherited anaemias. Lancet 2000;355:1169-75.

2. Taher A, Isma'eel H, Cappellini MD. Thalassemia intermedia: revisited. Blood Cells Mol Dis 2006;37:12-20.

3. Michel L, Auffray-Calvier E, Raoul S, et al. Spinal cord compression secondary to extra medullary hematopoiesis. Clin Neurol Neurosurg 2008;110:1073.

4. Moncef B, Hafedh J. Management of spinal cord compression caused by extramedullary hematopoiesis in beta-thalassemia. Intern Med 2008;47:1125-8.

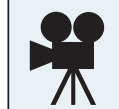

See video online of clonus in the patient's legs: www.cmaj.ca/cgi/content/full/cmaj.091427/DC1 